\title{
Identification of Type 2 Diabetes-associated combination of SNPs using Support Vector Machine
}

\author{
Hyo-Jeong Ban, Jee Yeon Heo, Kyung-Soo Oh and Keun-Joon Park*
}

\begin{abstract}
Background: Type 2 diabetes mellitus (T2D), a metabolic disorder characterized by insulin resistance and relative insulin deficiency, is a complex disease of major public health importance. Its incidence is rapidly increasing in the developed countries. Complex diseases are caused by interactions between multiple genes and environmental factors. Most association studies aim to identify individual susceptibility single markers using a simple disease model. Recent studies are trying to estimate the effects of multiple genes and multi-locus in genome-wide association. However, estimating the effects of association is very difficult. We aim to assess the rules for classifying diseased and normal subjects by evaluating potential gene-gene interactions in the same or distinct biological pathways.

Results: We analyzed the importance of gene-gene interactions in T2D susceptibility by investigating 408 single nucleotide polymorphisms (SNPs) in 87 genes involved in major T2D-related pathways in 462 T2D patients and 456 healthy controls from the Korean cohort studies. We evaluated the support vector machine (SVM) method to differentiate between cases and controls using SNP information in a 10-fold cross-validation test. We achieved a 65.3\% prediction rate with a combination of 14 SNPs in 12 genes by using the radial basis function (RBF)-kernel SVM. Similarly, we investigated subpopulation data sets of men and women and identified different SNP combinations with the prediction rates of $70.9 \%$ and $70.6 \%$, respectively. As the high-throughput technology for genome-wide SNPs improves, it is likely that a much higher prediction rate with biologically more interesting combination of SNPs can be acquired by using this method.
\end{abstract}

Conclusions: Support Vector Machine based feature selection method in this research found novel association between combinations of SNPs and T2D in a Korean population.

\section{Background}

It is estimated that by the year 2030, there will be $\sim 366$ million people affected by Type 2 diabetes (T2D) worldwide [1], with many of those affected lying in the middle to late adult years group [2]. T2D is genetically heterogeneous disease by the complex interplay of several environmental factors and susceptibility genes [3]. Singlenucleotide polymorphism (SNP) exhibits an abundant form of genetic variations. SNPs can be distinguished from other rare variations by more than $1 \%$ frequency in the human population when a single nucleotide replaces

\footnotetext{
*Correspondence: park-kj@cdc.go.kr

1 Division of Bio-Medical Informatics, Center for Genome Science, National Institute of Health, Korea Center for Disease Control and Prevention, 194, Tongil-Lo, Eunpyung-Gu, Seoul 122-701, Republic of Korea Full list of author information is available at the end of the article
}

one of the three nucleotides. The human genome contains about 10 30 million SNPs with an average SNP every 100 300 bases. More than 5 million human SNPs have been identified and the information is publicly available (NCBI dbSNP Build 129). A SNP in a protein coding sequence (CDS) can induce amino acid changes, resulting in functional changes in the protein. Some SNPs in a promoter region can effect transcriptional regulation, and a SNP in an intron region can affect the splicing or expression of the gene.

In recent years, genome-wide association studies (GWAS) have identified a large number of robust associations between genetic variation and complex human disease, such as Type 2 diabetes and rheumatoid arthritis [4]. These approaches have identified common genetic variants that are associated with the risk of more than 40 
diseases and human phenotypes [5]. In the T2D studies, candidate gene or genome-wide association approaches have suggested various putative T2D susceptibility SNP variants in various genes including TCF7L2, PPARG, KCNJ11, CDKN2A/B, FTO, CDKAL1 and so on [6-10]. But individual susceptibility of SNP variants may be disappointingly small or nowhere near enough to explain estimates of heritability [11]. One possible explanation for these weak relative risks and low attributable risks is that the risk may vary across different groups of clinically and biologically distinct T2D; further, analyzing T2D as a single disease may obscure the association with these risk factors. Another possible explanation is the effects of gene-gene (SNP-SNP) interactions. Most complex diseases result from the poorly understood interaction of genetic-genetic and genetic-environmental factors. The biological phenomenon associated with T2D that are modestly affected by a single SNP might be much greatly affected by a SNP in combination with additional SNPs in genes derived from the same or distinct biological pathways. In other words, it is difficult to identify diseaselinked variants that are too rare to be picked up by association methods and yet have risk alleles of sufficient effects to allow detection with the use of existing statistical strategies [12]. A marker strongly related to risk does not guarantee effective discrimination between cases and controls [13].

A goal of this research is to assess the rules for classifying the case (T2D) and control (non-T2D) groups along with considering the potential gene-gene (SNP-SNP) interactions. Since it is considered that the SNPs are less influential toward the onset or development of T2D than combinations of SNPs, our interest is specially focused on the classification of SNPs that can detect the putative effects of genetic interactions. Small effects that could, when combined, have a significant impact on someone's health including onset of T2D, thus to get overall view of risk, the effects of the individual SNPs have to be combined [11]. There are several researches designed to examine the effect of combined SNPs to disease risks. Some methods have used the multifactor dimensionality reduction (MDR) algorithm, which identifies all the possible combinations of SNPs from a set of given SNPs, and the combination of SNPs that optimally predicts the risk by minimizing the classification error of cases and controls is finally selected [14]. Goodman and colleagues formulated a polymorphism interaction analysis (PIA) method, which examines all the possible SNP combinations (similar to MDR) among 94 SNPs in 63 genes studied in 216 male colon cancer cases and 255 male controls. They employed two separate functions that cross-validate and minimize the false-positive results in the evaluation of SNP combinations to predict the risk of colon cancer [15].
In the present research, we analyzed the associations between the combination of SNPs and T2D using a Support Vector Machine (SVM) - a machine learning algorithm. Classification based on the SVM has several applications in bioinformatics and computational biology [16-20]. It has been widely used to predict protein secondary structures [21], solvent accessibility [22,23], protein-protein binding sites [24], remote protein homology detection [25], detection of non-coding RNA [26], protein domains [27], protein subcellular localization [2830], discrimination of outer membrane protein [31], and gene and tissue classification from the microarray expression data [32].

Several researchers have recently applied this powerful machine-learning algorithm--SVM--to the problem of identifying combinations of SNPs that can predict the susceptibility toward diseases. Listgarten and colleagues [33] considered the SNPs from 45 genes of potential relevance to breast cancer etiology in 174 patients as compared to the matched normal controls. They obtained an accuracy of $69 \%$ when using SVMs as the learning algorithm. They concluded that multiple SNPs from different genes over distant parts of the genome are better at identifying breast cancer patients than any single SNP alone. Waddell et al. (2005) have applied SVMs to predict the susceptibility to multiple myeloma. Their work provided $71 \%$ accuracy on a dataset containing 40 cases and 40 controls. Very recently, Uhmn et al. (2009) applied several machine learning techniques including SVM to predict patients' susceptibility to chronic hepatitis from SNPs [34].

In this research, we analyzed the importance of genegene interactions on T2D risk by investigating 408 SNPs from 87 genes involved in major T2D-related pathways in a sample of 462 T2D cases and 456 healthy population controls. We applied the SVM to discriminate cases and controls with SNP combination information by means of a 10-fold cross-validation test. From the target population, we achieved $65.3 \%$ prediction rate with a combination of 14 SNPs from 12 genes using the RBF (Radial Basis Function) kernel SVM. We also investigated men and women sub-population datasets using the same method, and identified some different combinations of SNPs with prediction rates of $70.9 \%$ and $70.6 \%$, respectively. For more precise identification of gene-gene interaction information in a biological manner, we may need more precise well-characterized sub-population datasets. In order to refine the genetic-environment relationship, more information is required in an epidemiological investigation. Besides existing statistical methods, we demonstrated the feasibility of incorporating SVM - a machine learning algorithm into case-control study. 


\section{Results}

\section{Case-Control Association Study}

For each SNP, the $p$-value was calculated based on a chisquared test. Based on the test results of 408 SNPs, 27 SNPs showed a significant genotype- or allele-based $p$ value $(<0.05)$ (Table 1$)$. The $-\log 10 p$-value from association result of SNPs was plotted in each chromosome and the significant SNPs are circle shape (Figure 1).

This candidate-gene based analysis may have some limitations to detect association from the small population size (462 cases and 456 controls) and the limited number of candidate genes (87 putative T2D-related genes). The result of this classical case-control association study may need a further replication study with a large independent target population of cases and controls for establishing the credibility of a genotype-phenotype association. We used this classical association study result in the process of sub-dataset filtering based on the genotype-based $p$ value range (Table 2 ).

\section{Combination of SNPs}

We performed SVM training and test analysis to find the best combination of SNPs. The prediction rates were determined by the SVM classifier that discriminated the case-control SNP genotype vectors. At first, we acquired $63.6 \%$ of the overall accuracy with the entire 408 SNP dataset, but we found that the p-value-based filtering method is useful for obtaining a better prediction rate. The prediction rate of a higher $p$-value SNP dataset (Table 1) did not show the best result (57.6\%). This effect might be attributed to the different effects between a single SNP and within a combination of SNPs. This $p$-valuebased filtering can reduce the search space for gene-gene interactions from a very large number of all possible combinations of SNPs to a manageable dataset.

Another reason is the limitation of the forward selection method to find the best combination of SNPs. The entire set of 408 SNPs may contain noise SNPs for forward selection, and some useful SNPs in the ideal combination may be removed from the very restricted $p$-valuebased filtered SNP dataset (e.g., 24 SNPs with $p<0.05$ ).

The best prediction rate of the SVM classifier with a RBF kernel function was $65.3 \%$ with 14 SNPs including a combination from the 240 SNPs with $\mathrm{p}<0.6$ (Table 2 and Table 3). In table 3, rs343 was reported the association with T2D [35], and two of SNPs (rs2070011 and rs2243250) were reported with not T2D but myocardial infarction [36,37]. Furthermore, sub-population datasets of men and women with the RBF kernel, which were designed to discriminate case and control, yielded slightly better prediction rates of $70.9 \%$ and $70.6 \%$, respectively, than that of the total population dataset (Table 4, Table 5 and Table 6). These prediction rates are almost similar with other previous studies using SVM, for example 69\% of Listgarten and colleagues [33], 67.5\% of Uhmn et al. [34], or 53\% of Schwender et al. [38]. But, these previous works used different disease samples and different crossvalidation test, thus it is difficult to compare these prediction rates directly. Considering other environmental and genetic factors involved in the development of T2D, the prediction performance was reasonably acceptable. It may be presumed that including other important genes and clinical factors including family medical history, we would obtain more improved prediction rate in the future. Different results between the entire target popula-

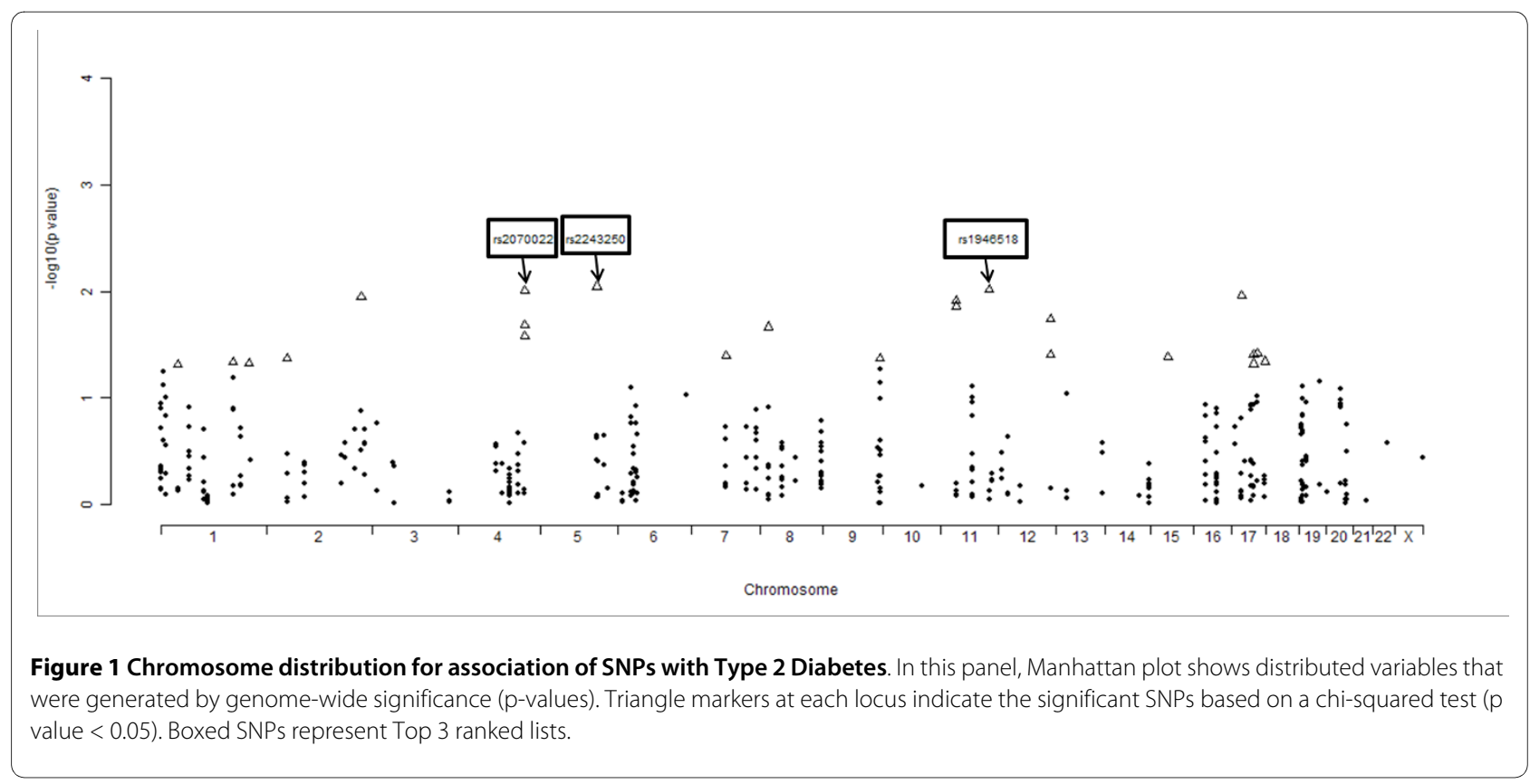


Table 1: Summary of the association study (genotype- or allele-based $\mathbf{p}<0.05$ )

\begin{tabular}{|c|c|c|c|c|c|c|c|}
\hline \multirow[t]{2}{*}{ Gene } & \multirow{2}{*}{$\begin{array}{c}\text { dbSNP } \\
\text { ID(b129) }\end{array}$} & \multirow{2}{*}{$\begin{array}{c}\text { Chromoso } \\
\text { me }\end{array}$} & \multirow[t]{2}{*}{ Location } & \multirow[t]{2}{*}{ Region* } & \multirow[t]{2}{*}{ Alleles* } & \multicolumn{2}{|l|}{$x^{2 p-v a l u e}$} \\
\hline & & & & & & Genotype & Allele \\
\hline SELE & rs4786 & 1 & 167958756 & 3'UTR & $G>A$ & 0.0462 & 0.1299 \\
\hline IL10 & rs1554286 & 1 & 205010856 & intron & $\mathrm{T}>\mathrm{C}$ & 0.0470 & 0.0170 \\
\hline CAP1 & rs16837478 & 1 & 40207033 & 3'UTR & $C>A$ & 0.0489 & 0.5850 \\
\hline VAMP3 & rs707457 & 1 & 7753651 & nearGene-5 & $\mathrm{G}>\mathrm{T}$ & 0.0755 & 0.0299 \\
\hline VAMP3 & novel & 1 & 7775035 & 3'UTR & $A>G$ & 0.0567 & 0.0220 \\
\hline SLC11A1 & novel & 2 & 219084963 & exon & $\mathrm{G}>\mathrm{A}$ & 0.0113 & 0.0229 \\
\hline $\mathrm{RHOQ}$ & rs17038378 & 2 & 46661749 & nearGene-3 & $W>D$ & 0.0426 & 0.1773 \\
\hline FGA & rs2070022 & 4 & 155724398 & 3'UTR & $C>T$ & 0.0099 & 0.0083 \\
\hline FGA & rs6050 & 4 & 155727040 & exon & $A>G$ & 0.0261 & 0.0077 \\
\hline FGA & rs2070011 & 4 & 155731347 & nearGene-3 & $A>G$ & 0.0208 & 0.0053 \\
\hline IL4 & rs2243250 & 5 & 132037053 & nearGene-5 & $\mathrm{T}>\mathrm{C}$ & 0.0090 & 0.0025 \\
\hline SOD2 & rs5746136 & 6 & 160023074 & intron & $G>A$ & 0.0948 & 0.0348 \\
\hline CD36 & rs3211908 & 7 & 80131852 & intron & $C>T$ & 0.0402 & 0.1445 \\
\hline LPL & rs343 & 8 & 19855067 & intron & $C>A$ & 0.0215 & 0.0075 \\
\hline RAPGER1 & rs875968 & 9 & 133461085 & intron & $\mathrm{G}>\mathrm{A}$ & 0.0423 & 0.1247 \\
\hline IL18 & rs1946518 & 11 & 111540668 & nearGene-5 & $\mathrm{T}>\mathrm{G}$ & 0.0097 & 0.1591 \\
\hline CAT & rs17886119 & 11 & 34417280 & intron & $C>T$ & 0.0138 & 0.2849 \\
\hline CAT & rs1408034 & 11 & 34432364 & intron & $C>T$ & 0.0123 & 0.2745 \\
\hline TCF1 & rs1169288 & 12 & 119901033 & exon & $\mathrm{T}>\mathrm{G}$ & 0.0391 & 0.6975 \\
\hline TCF1 & rs2464196 & 12 & 119919810 & exon & $\mathrm{T}>\mathrm{C}$ & 0.0181 & 0.9747 \\
\hline SNAP23 & rs9302112 & 15 & 40607743 & intron & $\mathrm{T}>\mathrm{C}$ & 0.0414 & 0.0842 \\
\hline $\mathrm{ACE}$ & rs4362 & 17 & 58927493 & exon & $C>T$ & 0.0384 & 0.0270 \\
\hline NOS2A & rs2297518 & 17 & 23120724 & exon & $\mathrm{G}>\mathrm{A}$ & 0.0109 & 0.5354 \\
\hline STXBP4 & rs9902718 & 17 & 50416621 & intron & $\mathrm{T}>\mathrm{C}$ & 0.0478 & 0.0156 \\
\hline STXBP4 & rs10468513 & 17 & 50417902 & intron & $\mathrm{C}>\mathrm{A}$ & 0.0478 & 0.0156 \\
\hline STXBP4 & rs11658717 & 17 & 50431985 & intron & $A>G$ & 0.0396 & 0.0128 \\
\hline ASPSCR 1 & novel & 17 & 77562902 & intron & $G>T$ & 0.0456 & 0.0511 \\
\hline
\end{tabular}

* Alleles and region for which the effect is estimated refer to the positive strand based on NCBI build 36

tion and men or women sub-population may arise from the effect of the dataset's size or the well-characterized sub-population grouping.

We could not find better prediction results by the above $p$-value-based filtering as that in Table 2 with men and women sub-population datasets. This result with a slightly improved prediction rate may arise from the effect of a smaller size of sub-datasets ( $n=405$ and 513) or the effect of well-characterized (gender-distinguished) sub-population datasets.

\section{Protein-Protein Interaction Information}

On the basis of the results of the combinations of SNPs, we attempted to find any biological information; one of the results is the protein-protein interaction (PPI) network (Figure 2), which was constructed from the results of the combinations of SNPs. Each set of the SNP genotype data was not acquired from the fine mapping association study; therefore, direct SNP-SNP interaction or SNP analysis focused on each promoter SNP, intron SNP, or exon SNP is difficult. This is the reason why we carried out the analysis at the protein (gene) level in this research (not the SNP level).

The genomenetwork platform http://genomenetwork.nig.ac.jp provides protein-protein interaction network from the $\mathrm{Y} 2 \mathrm{H}$ experimental data and the public databases (BIND, MINT and HPRD). Also, it has interac- 
Table 2: Prediction rate of combinations of SNPs with genotype-based p-value filtering

\begin{tabular}{|c|c|c|c|c|c|}
\hline \multirow[t]{2}{*}{ p-value range } & \multirow[t]{2}{*}{ No. of SNPs [1] } & \multicolumn{3}{|c|}{ Prediction rate } & \multirow[t]{2}{*}{ No. of SNPs [2] } \\
\hline & & Overall & Sensitivity & Specificity & \\
\hline$<0.05$ & 24 & 0.576 & 0.545 & 0.607 & 4 SNPs \\
\hline$<0.1$ & 40 & 0.600 & 0.593 & 0.607 & 6 SNPs \\
\hline$<0.2$ & 92 & 0.632 & 0.660 & 0.603 & 10 SNPs \\
\hline$<0.3$ & 129 & 0.642 & 0.630 & 0.654 & 12 SNPs \\
\hline$<0.4$ & 169 & 0.642 & 0.630 & 0.654 & 12 SNPs \\
\hline$<0.5$ & 199 & 0.651 & 0.571 & 0.732 & 13 SNPs \\
\hline$<0.6$ & 240 & 0.653 & 0.567 & 0.739 & 14 SNPs \\
\hline$<0.7$ & 290 & 0.651 & 0.610 & 0.693 & 12 SNPs \\
\hline$<0.8$ & 335 & 0.636 & 0.721 & 0.550 & $7 \mathrm{SNPs}$ \\
\hline$<0.9$ & 372 & 0.636 & 0.721 & 0.550 & 7 SNPs \\
\hline$<1.0$ & 408 & 0.636 & 0.721 & 0.550 & 7 SNPs \\
\hline
\end{tabular}

[1] No. of SNPs for each genotype-based $p$-value range; [2] No. of SNPs for each combination

tion property and gene annotation information. We obtained gene interaction information from PPI database. Circles (proteins) are included in the results of the combination of SNPs, and circles are collected from the entire PPI information database to connect with the squares. The construction of an indirect PPI network of two proteins is unnecessary from the biological viewpoint; therefore, we permit only two or fewer proteins (squares) between two proteins (circles) in Figure 2. We could easily find the same proteins among the target population datasets and the target-population-specific proteins in these three PPI networks. PPI network of Figure 2a contains 7 genes from the SNP combination result of 12 genes (14 SNPs) in Table 3. Other PPI network of Figure $2 \mathrm{~b}$ and $2 \mathrm{c}$ contains 4 genes and 6 genes from the SNP combination results of men and women sub-data sets in

Table 3: List of 14 SNPs for the best combination of SNPs

\begin{tabular}{|c|c|c|c|c|c|}
\hline Gene & dbSNP ID & Chromosome & Location & Region & Allele \\
\hline IRS1 & rs6436635 & 2 & 227373922 & nearGene-5 & $G>A$ \\
\hline SLC11A1 & Novel* & 2 & 17459455 & exon & $\mathrm{G}>\mathrm{A}$ \\
\hline FGA & rs2070011** & 4 & 155731347 & 5'UTR & $A>G$ \\
\hline SPP1 & rs2853749\# & 4 & 89116838 & intron & $\mathrm{C}>\mathrm{T}$ \\
\hline IL4 & rs2243250 & 5 & 132037053 & nearGene-5 & $\mathrm{T}>\mathrm{C}$ \\
\hline IL4 & rs56279116 & 5 & 132038071 & exon & $G>A$ \\
\hline PPARD & rs9658173 & 6 & 35502649 & 3'UTR & $G>A$ \\
\hline LPL & rs343\#\# & 8 & 19855067 & intron & $C>A$ \\
\hline TCF1 & rs2464196 & 12 & 119919810 & exon & $\mathrm{T}>\mathrm{G}$ \\
\hline$A C E$ & rs13306087 & 17 & 58910142 & exon & $\mathrm{G}>\mathrm{A}$ \\
\hline ASPSCR1 & Novel* & 17 & 77562902 & intron & $\mathrm{G}>\mathrm{T}$ \\
\hline NOS2A & rs9282801 & 17 & 23120600 & intron & $G>T$ \\
\hline INSR & rs2303672 & 19 & 7119405 & intron & $A>G$ \\
\hline INSR & rs3745548 & 19 & 7103703 & intron & $A>G$ \\
\hline
\end{tabular}

* Novel SNP in KHGS does not exist on dbSNP database.

** rs2070011 is associated with myocardial infarction [36]

\#rs2243250 is associated with myocardial infarction [37]

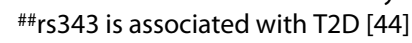


Table 4: Prediction rates of the SVM classifiers with different target populations

\begin{tabular}{ccccc}
\hline Target population & Sensitivity & Specificity & Overall accuracy & $\begin{array}{c}\text { No. of SNPs for each } \\
\text { combination }\end{array}$ \\
\hline Total & 0.567 & 0.739 & 0.653 & 14 SNPs \\
Men\# & 0.714 & 0.704 & 0.709 & 12 SNPs \\
Women $\#^{\#}$ & 0.715 & 0.696 & 0.706 & 19 SNPs \\
\hline
\end{tabular}

\# 199 Cases; 206 Controls

\#\#263 Cases; 250 Controls

Table 5 and 6, respectively. IL4 (interleukin 4) gene is the common gene among these three PPI networks and IL4, INSR, and IRS1 genes are common between Figure 2a (total population set) and 2c (women sub-population set).

\section{Discussion}

It is widely agreed that complex diseases are typically caused by the joint effects of multiple genetic variations instead of a single genetic variation. The gene-gene (epistatic) interactions of SNPs are believed to be very important in determining individual susceptibility to complex diseases. Thus, it is desirable to develop an effective method to search gene-gene interactions in human genome data. Recently, some computational methods have been proposed to address this issue using Multifactor Dimensional Reduction (MDR), or machine learning algorithms [39]. To study complex disease such as T2D, it is possible that many genes contribute to a T2D by their interaction with other genes, while main effects of the individual gene may be small or absent. Therefore, we developed the method that specifically designed to detect multiple disease SNPs, possibly on different chromosomes using SVM. This approach could be useful for identification of potential disease markers which geno- type patterns are significantly associated with a high susceptibility.

This analysis includes the SNPs information of 87 T2Drelated genes from fatty acid binding/translocation, GLUT4 translocation, and insulin signal pathways. A primary function of insulin is to stimulate the transport of glucose into target tissue, prominent among which are skeletal muscle, cardiac muscle, and adipose tissue. Insulin achieves this effect by inducing the translocation of GLUT4 glucose transporters from an intracellular vesicular compartment to the plasma membrane. Under basal condition, GLUT4 cycles between this intracellular compartment and the plasma membrane. SNAP23 is required for insulin-induced GLUT4 translocation to the plasma membrane and that it mediates the formation of a complex between syntaxin 4 and VAMP2 [40].

T2D results from impairment in both insulin sensitivity and insulin secretion. Several genes have been implicated that might contribute significantly to the risk of T2D, including TCF7L2, PPARG, KCNJ11, CDKN2A/B and so on $[6,8-10]$. T2D is one of the typical complex disease (polygenic disorder), which likely associated with the effects of multiple genes (SNPs) in combination with lifestyle and other environmental factors. In this research,

Table 5: List of 12 SNPs for the best combination of SNPs (men)

\begin{tabular}{|c|c|c|c|c|c|}
\hline Gene & dbSNP ID & Chromosome & Location & Region & Allele \\
\hline LEPR & rs1805134 & 1 & 65839697 & exon & $A>G$ \\
\hline PRKCZ & rs3795277 & 1 & 1970978 & nearGene-5 & $A>C$ \\
\hline PPARG & rs13306747 & 3 & 12433274 & exon & $C>G$ \\
\hline FABP2 & rs1799883 & 4 & 120461350 & exon & $G>A$ \\
\hline UCP1 & novel & 4 & 141848403 & promoter & $G>A$ \\
\hline IL4 & novel & 5 & 132038071 & exon & $\mathrm{G}>\mathrm{A}$ \\
\hline LPL & rs3208305 & 8 & 19867928 & 3'UTR & $A>T$ \\
\hline LPL & rs13702 & 8 & 19868772 & 3'UTR & $A>G$ \\
\hline STXBP4 & rs1894936 & 17 & 50475854 & intron & $A>G$ \\
\hline LDLR & rs6413504 & 19 & 11102915 & intron & $A>G$ \\
\hline LDLR & rs1433099 & 19 & 11103658 & 3'UTR & $G>A$ \\
\hline ACAS2 & novel & 20 & 32926612 & promoter & $G>T$ \\
\hline
\end{tabular}


Table 6: List of 19 SNPs for the best combination of SNPs (women)

\begin{tabular}{|c|c|c|c|c|c|}
\hline Gene & dbSNP ID & Chromosome & Location & Region & Allele \\
\hline CAP1 & rs16837478 & 1 & 40207033 & 3'UTR & $\mathrm{C}>\mathrm{A}$ \\
\hline IL10 & rs1554286 & 1 & 203332628 & intron & $\mathrm{T}>\mathrm{C}$ \\
\hline LEPR & rs13306523 & 1 & 65603011 & 5'UTR & $C>T$ \\
\hline MTHFR & rs2274976 & 1 & 11785193 & exon, nearGene-3 & $\mathrm{G}>\mathrm{A}$ \\
\hline SELE & rs5355 & 1 & 166427528 & exon & $C>T$ \\
\hline VAMP3 & novel & 1 & 7775035 & 3'UTR & $A>G$ \\
\hline IRS1 & rs6436635 & 2 & 227373922 & nearGene-5 & $\mathrm{G}>\mathrm{A}$ \\
\hline IRS1 & rs1801278 & 2 & 227486049 & exon & $\mathrm{G}>\mathrm{A}$ \\
\hline NEUROD1 & rs1801262 & 2 & 182368961 & exon & $\mathrm{G}>\mathrm{A}$ \\
\hline SLC11A1 & novel & 2 & 219084963 & exon & $\mathrm{G}>\mathrm{A}$ \\
\hline GPX1 & rs3811699 & 3 & 49371364 & nearGene-5 & $A>G$ \\
\hline IL4 & novel & 5 & 132038071 & exon & $\mathrm{G}>\mathrm{A}$ \\
\hline SLC27A4 & rs17848327 & 9 & 128192130 & intron & $\mathrm{G}>\mathrm{A}$ \\
\hline MMP2 & rs17859889 & 16 & 54077202 & intron & $\mathrm{C}>\mathrm{T}$ \\
\hline MMP2 & rs17860021 & 16 & 54097255 & 3'UTR & $\mathrm{G}>\mathrm{A}$ \\
\hline ASPSCR1 & novel & 17 & 77528191 & nearGene-5 & $\mathrm{G}>\mathrm{C}$ \\
\hline STXBP4 & rs10468513 & 17 & 50417902 & nearGene-5 & $\mathrm{C}>\mathrm{A}$ \\
\hline STXBP4 & rs11658717 & 17 & 50431985 & intron & $A>G$ \\
\hline INSR & novel & 19 & 7135243 & intron & $\mathrm{G}>\mathrm{C}$ \\
\hline
\end{tabular}

we analyzed the candidate genes data set, thus the result does not contain these known significant SNP markers, including TCF7L2, PPARG, KCNJ11 and so on.

The result from Table 1 (all cases, combined versus controls) indicated weak associations with the risk factors investigated. This led us to stratify by sub-grouping by gender to see whether some potential associations may have been obscured by considering T2D as one disease. In this research, we first made two subpopulation data sets by gender (Table 4, and Figure 2). Epidemiological evidence suggests that sex differences exist in T2D. The prevalence of T2D is higher in men than women. Globally, diabetes prevalence is similar in men and women but it is slightly higher in men $<60$ years of age and in women at older ages [1]. This difference may possibly result from the differences in insulin sensitivity and regional body fat deposition $[41,42]$.

Yeh et al. [43] used a conditional knockout strategy to generate androgen receptor (AR) knockout mice to study the relationship between androgen-AR and insulin sensitivity, and Lin et al. reported the influences of loss of AR on insulin and leptin resistance. Loss of AR may contribute to an increase of leptin levels and leptin resistance, which may play important roles for the development of obesity and insulin resistance. Important factors such as age at onset of T2D can also be incorporated in the modeling to further partition phenotypic variation or for defining subtypes of the phenotype.
As high-throughput technology for genome-wide SNP genotyping ( $500 \mathrm{~K}$ or 1 mega) improves and as more SNPs are identified, it is likely that much higher prediction rate will be achieved and a useful clinical system developed. For the biologically more precise identification of gene-gene interaction's effect for T2D, we may need more precise well characterized subpopulation data sets and more powerful computational power and method and so on. Besides existing statistical methods, we demonstrated the feasibility of incorporating SVM - a machine learning algorithm into case-control study. We plan to develop the method using machine learning algorithm in the future to search gene-gene interactions for our new Genome-Wide Association Study (GWAS) data [44].

\section{Conclusions}

We have found novel association between combinations of SNPs and T2D in a Korean population. We proposed gene-gene interaction considering candidate genes association study using SVM based feature selection method in this research.

\section{Methods}

\section{Data and Data Preprocessing}

Our dataset consists of 408 SNP data distributed over putative 87 T2D-related genes in 462 cases (patients) and 456 normal controls. The T2D cases, confirmed and 


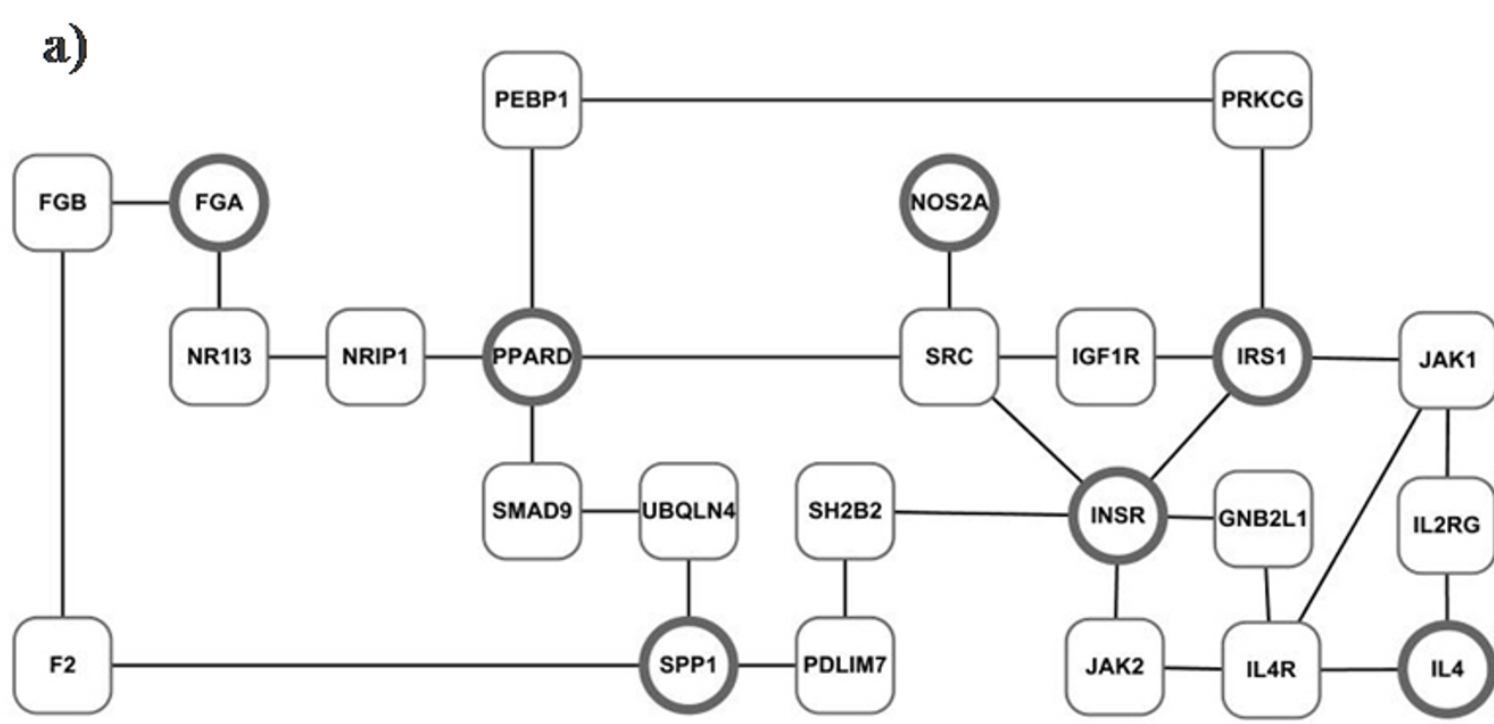

b)

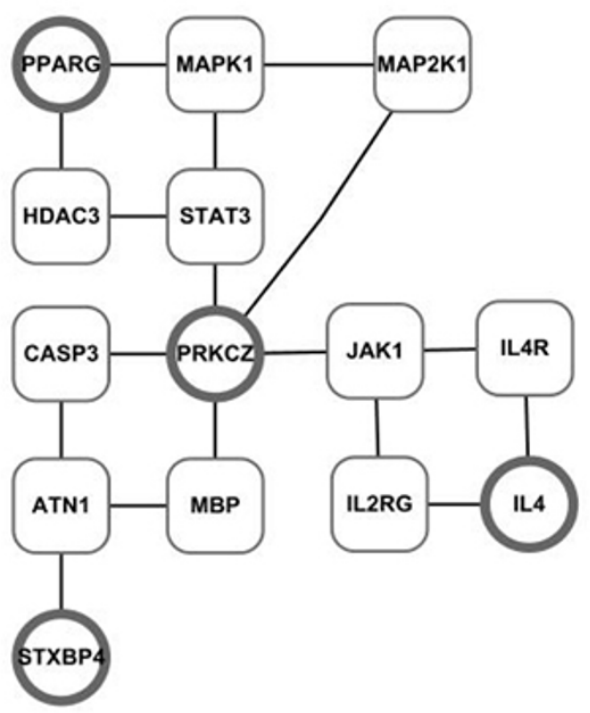

c)

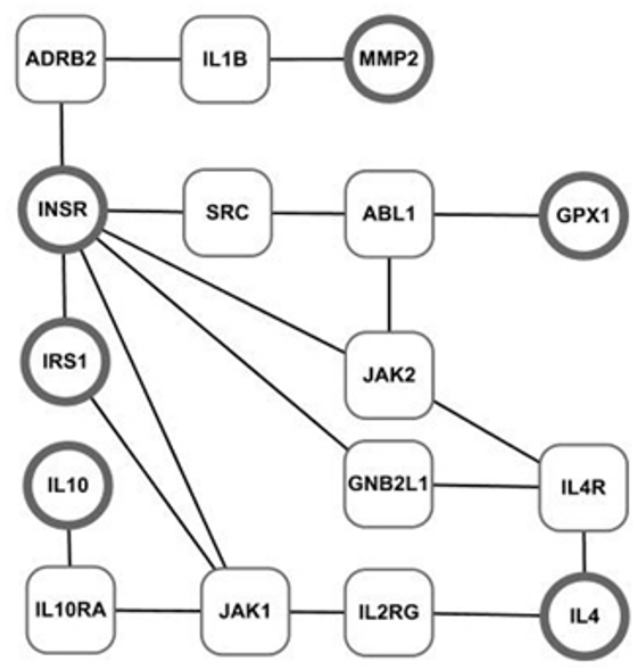

Figure 2 PPI network from the SNP combination of (a) total population set, (b) men sub-population set, and (c) women sub-population set. The largest PPI network at each population set was constructed using PPI information database http://genomenetwork.nig.ac.jp. Circles are included in the best combination of SNPs from SVM results and square proteins are included to construct circle-circle connected proteins network.

diagnosed in the Ansan and Ansung cohort study area, were identified from the Korean Health and Genome Study (KHGS). The Ansan area primary represents an urban community, whereas the Ansung area represents a rural community in Korea. These two cohort studies include information on 87 T2D-related genes from fatty acid binding/translocation, GLUT4 (insulin-responsive glucose transporter 4) translocation, and insulin signal pathways. We selected these three pathways with reference survey [45-48]. Among identified polymorphisms,
408 SNPs were selected based on location (CDS (protein coding sequence), intron, UTR (Untranslated region), promoter etc), frequency, linkage disequilibrium (LD) status and so on. The number of SNPs occurring in the CDS, intron, UTR, near gene region (promoter) and intergenic region was $77,169,53,73$ and 36 , respectively (additional file 1). In this research, 462 cases were defined from two cohort studies as T2D subjects according to the World Health Organization (WHO) criteria. The 456 unrelated normal control people have no history of T2D, 
no first-degree relatives with T2D, fasting plasma glucose level less than $126 \mathrm{mg} / \mathrm{dL}$, plasma glucose level $120 \mathrm{~min}$ after glucose ingestion of less than $140 \mathrm{mg} / \mathrm{dL}$, and HbA1C level (glycosylated hemoglobin) of less than 5.8\%. Further, the normal control people do not have a history of diabetes, hypertension, and dyslipidemia. In this study, all the people of case and control were more than 60 years of age.

For each SNP, the $p$-value was calculated based on a chi-squared test without adjustment for other confounding variables (Table 1). In this paper, we applied SVM to predict the susceptibility to T2D using SNP genotype data. From the view point of binary classification, we treated T2D cases as positive samples and controls as negative samples, and we used SNP variants as categorical features that have three possible genotype values at a locus. Usually, a SNP genotype is represented by a number that matches 1,2 , or 3 , where 1 represents the homozygous site with a major allele, 2 represents a heterozygous site, and 3 represents a homozygous site with a minor allele [33]. Waddell et al. (2005) have applied SVMs to predict the susceptibility to multiple myeloma using $-1,0,1$, where 0 represents a heterozygous site and -1 and 1 arbitrarily represent homozygous sites. The preprocessing method used in this research was the same as that used by Listgarten et al. (2004) [33].

\section{Support Vector Machine}

A SVM is a learning algorithm that learns a classifier from a set of positively and negatively labeled training vectors, which can be used to classify new unlabelled test samples. The SVM learns the classifier by mapping the input training samples into a possibly high-dimensional feature space, and seeking a hyperplane in this space that separates the two types of examples with the largest possible margin, i.e., the distance to the nearest points. If the training set is not linearly separable, the SVM finds a hyperplane that optimizes the trade-off between good classification and large margin with a slack variable and kernel trick. For an actual implementation, we used the freely downloadable SVM-light package [49]. We tested linear, polynomial, and radial basis function (RBF) kernels with various parameters, and the final results were acquired with the RBF kernel and parameter gamma 1 that yielded the best prediction rate. We treated T2D cases as positive samples and controls as negative samples, and used SNP genotypes as categorical features. We adopted SVM to discriminate T2D cases against controls in this research.

\section{Feature Selection}

For large datasets, an exhaustive consideration of all the possible SNP combinations can become computationally infeasible. Therefore, we employed a feature selection procedure to find the best putative combination of SNPs according to forward selection for handling datasets with a genotype of SNPs. In population studies, this kind of selection of informative SNPs was usually developed for population identification [50]. In this work, forward selection was started by first selecting the SNP feature that yielded the best fit for the independent test set using SVM training and test at a time. This SNP feature was used to test all the combinations with the remaining 407 (408 - 1) SNPs in order to find the best pair of SNP features. This process continues step by step until increasing the size of the current subset leads to a lower overall accuracy. We adopted the 10 -fold cross-validated classification accuracy for the selection criteria in this work. The requirement of the best prediction rate at each step yields the highest overall accuracy with regard to both sensitivity and specificity $(\geq 0.45)$. The purpose of this requirement is to avoid the extremely low sensitivity or specificity with the highest overall accuracy.

Since we have a relatively small number of people (462 cases and 456 controls) in our dataset, it is expected that training with the complete set of 408 SNP features may cause overfitting. Hence, we performed forward selection with SNP genotype features to find a good smaller feature set (a combination of SNPs). Note that forward selection does not necessarily find the best combination of SNPs. However, it usually results in a combination that comes close to the optimum solution, and it needs relatively less computational complexity. If we have a smaller datasets and a more powerful computer, step-wise feature selection may be a better method than the forward selection method in this study.

\section{Cross-Validation Test}

The prediction rates of the SVM classifiers were examined by the 10-fold cross-validation test, where each case and control dataset is randomly divided into 10 subsets of approximately the same size. The SVM classifiers were trained 10 times, leaving out one of the subsets from the training each time. This single subset was used to estimate the prediction rate of the trained SVM classifier. The prediction rate of the SVM classifiers was evaluated using three measures, namely, sensitivity, specificity, and overall accuracy.

$$
\begin{aligned}
\text { sensitivity } & =\frac{T P}{T P+F N} \\
\text { specificity } & =\frac{T N}{T N+F P} \\
\text { overall accuracy } & =\frac{T P+T N}{T P+T N+F P+F N}
\end{aligned}
$$


where $T P, F P, T N$, and $F N$ refer to the number of true positives, false positives, true negatives, and false negatives statuses (case or control), respectively. Sensitivity measures the ability to correctly predict $\mathrm{T} 2 \mathrm{D}$ cases, while specificity measures the ability for correctly reject controls. The kernel functions and parameters for the classification algorithms were optimized during the 10-fold cross-validation tests, while avoiding overfitting problems.

\section{Additional material}

\section{Additional file 1 General information of $\mathbf{4 0 8}$ SNPs data set. 408 SNPs} were selected 87 T2D-related genes from fatty acid binding/translocation, GLUT4 (insulin-responsive glucose transporter 4) translocation and insulin signal pathways. Excel file shows the general SNP information including SNP ID (rs number), chromosomal location information and so on.

\section{Authors' contributions}

HJB drafted the manuscript, participated in the design the study, and contributed to the statistical and functional analysis. JYH co-designed the study, performed the SVM machine learning and statistical analysis, KSO helped draft the manuscript and critically reviewed the manuscript. KJP investigated and guided the study project and drafted the manuscript. All authors read and approved the final manuscript.

\section{Acknowledgements}

This work was supported by an intramural grant from the Korea National Institute of Health, Korea Center for Disease Control and Prevention, Republic of Korea (4845-301-210). This work was also supported by Korea Research Environment Open NETwork.

\section{Author Details}

Division of Bio-Medical Informatics, Center for Genome Science, National Institute of Health, Korea Center for Disease Control and Prevention, 194, Tongil-Lo, Eunpyung-Gu, Seoul 122-701, Republic of Korea

Received: 23 June 2009 Accepted: 23 April 2010

Published: 23 April 2010

\section{References}

1. Wild S, Roglic G, Green A, Sicree R, King H: Global prevalence of diabetes: estimates for the year 2000 and projections for 2030. Diabetes Care 2004, 27:1047-1053.

2. Association AD: Economic consequences of diabetes mellitus in the U.S. in 1997. American Diabetes Association. Diabetes Care 1998, 21:296-309.

3. Owen KR, McCarthy MI: Genetics of type 2 diabetes. Curr Opin Genet Dev 2007, 17(3):239-244.

4. Hunter DJ, Kraft P: Drinking from the fire hose - statistical issues in genome wide association studies. N Engl J Med 2007, 357:436-439.

5. Kraft P, Hunter DJ: Genetic Risk Prediction-Are We There Yet? N Eng/J Med 2009, 360(17):1701-1703

6. Altshuler D, Hirschhorn JK, Klannemark M, Lindgren CM, VohI MC, Nemesh J, Lane CR, Schaffner SF, Bolk S, Brewer C, et al:: The common PPARgamma Pro12Ala polymorphism is associated with decreased risk of type 2 diabetes. Nat Genet 2000, 26(1):76-80.

7. Consortium WTCC: Genome-wide association study of 14,000 cases of seven common diseases and 3,000 shared controls. Nature 2007, 447:661-678.

8. Gloyn AL, Weedon MN, Owen KR, Turner MJ, Knight BA, Hitman G, Walker M, Levy JC, Sampson M, Halford S, et al:: Large-scale association studies of variants in genes encoding the pancreatic beta-cell KATP channel subunits Kir6.2 (KCNJ11) and SUR1 (ABCC8) confirm that the KCNJ11 E23K variant is associated with type 2 diabetes. Diabetes 2003, 52(2):568-572.
9. Grant SF, Thorleifsson G, Reynisdottir I, Benediktsson R, Manolescu A, Sainz J, Helgason A, Stefansson H, Emilsson V, Helgadottir A, et al:: Variant of transcription factor 7-like 2 (TCF7L2) gene confers risk of type 2 diabetes. Nat Genet 2006, 38(3):320-323.

10. Scott L, Mohlke KL, Bonnycastle LL, Willer CJ, Li Y, Duren WL, Erdos MR, Stringham HM, Chines PS, Jackson AU, Prokunina-Olsson L, et al:: A genome-wide association study of type 2 diabetes in Finns detects multiple susceptibility variants. Science 2007, 316(5829):1341-1345.

11. Masher B: Personal genomes: The case of the missing heritability. Nature 2008, 456:18-21.

12. Hardy J, Singleton A: Genomewide Association Studies and Human Disease. NEngl J Med 2009, 360:1759-1768.

13. Jakobsdottir J, Gorin MB, Conley YP, Ferrell RE, Weeks DE: Interpretation of genetic association studies: markers with replicated highly significant odds ratios may be poor classifiers. PLos Genet 2009, 5(2):e1000337.

14. Hahn LW, Ritchie MD, Moore JH: Multifactor dimensionality reduction software for detecting gene-gene and gene-environment interactions. Bioinformatics 2003, 19:376-382.

15. Goodman JE, Mechanic LE, Luke BT, Ambs S, Chanock S, Harris CC: Exploring SNP-SNP interactions and colon cancer risk using polymorphism interaction analysis. Int J Cancer 2006, 118(7):1790-1797.

16. Kecman V: Learning and Soft Computing, Support Vector machines, Neural Networks and Fuzzy Logic Models. The MIT Press, Cambridge, $\mathrm{MA} ; 2001$

17. Khandoker AH: Support Vector Machines for Automated Recognition of Obstructive Sleep Apnea Syndrome from ECG Recordings. IEEE Trans Inf Technol Biomed 2009, 13(1):37-48.

18. Sloin A, Burshtein D: Support Vector Machine Training for Improved Hidden Markov Modeling. IEEE trans signal process 2008, 56(1):172.

19. Wang LP: Support Vector Machines: Theory and Application. Springer, Berlin; 2005

20. Wang LP, Fu XJ: Data Mining with Computational Intelligence. Springer, Berlin; 2005.

21. Nguyen MN, Rajapakse JC: Two-stage multi-class support vector machines to protein secondary structure prediction. Pac Symp Biocomput 2005, 10:346-357.

22. Yuan Z, Burrage K, Mattick JS: Prediction of protein solvent accessibility using support vector machines. Proteins 2002, 48:566-570

23. Kim H, Park H: Prediction of protein relative solvent accessibility with support vector machines and long-range interaction $3 \mathrm{D}$ local descriptor. Proteins 2004, 54(3):557-562.

24. Bradford JR, Westhead DR: Improved prediction of protein-protein binding sites using a support vector machines approach. Bioinformatics 2005, 21:1487-1494.

25. Busuttil S, Abela J, Pace GJ: Support vector machines with profile-based kernels for remote protein homology detection. Genome Inform 2004, 15(2):191-200.

26. Wang C, Ding C, Meraz RF, Holbrook SR: PSoL: A positive sample only learning algorithm for finding non-coding RNA genes. Bioinformatics 2006, 22:2590-2596.

27. Vlahovicek K, Kajan L, Agoston V, Pongor S: The SBASE domain sequence resource, release 12: Prediction of protein domain-architecture using support vector machines. Nucleic Acids Res 2005, 33:D223-225

28. Hua S, Sun Z: Support vector machine approach for protein subcellular localization prediction. Bioinformatics 2001, 17:721-728.

29. Nair R, Rost B: Mimicking cellular sorting improves prediction of subcellular localization. J Mol Biol 2005, 348:85-100.

30. Park K-J, Kanehisa M: Prediction of protein subcellular locations by support vector machines using compositions of amino acid pairs. Bioinformatics 2003, 19:1656-1663.

31. Park K-J, Gromiha MM, Horton P, Suwa M: Discrimination of outer membrane proteins using support vector machines. Bioinformatics 2005, 21(23):4223-4229.

32. Brown MPS, Grundy WN, Lin D, Cristianini N, Sugnet CW, Furey TS, Ares M, Haussler D: Knowledge-based analysis of microarray gene expression data by using support vector machines. Proc Natl Acad Sci USA 2000 97:262-267

33. Listgarten J, Damaraju S, Poulin B, Cook L, Dufour J, Driga A, Mackey J, Wishart D, Greiner R, Zanke B: Predictive models for breast cancer susceptibility from multiple single nucleotide polymorphism. Clin Cancer Res 2004, 10:2725-2737. 
34. Uhmn S, Kim D-H, Ko Y-W, Cho S, Cheong J, Kim J: A study on application of single nucleotide polymorphism and machine learning techniques to diagnosis of chronic hepatitis. Expert Systems 2009, 26:60-69.

35. Cho YS, Go MJ, Han HR, Cha SH, Kim HT, Min H, Shin HD, Park C, Han BG, Cho $\mathrm{NH}$, et al:: Association of lipoprotein lipase (LPL) single nucleotide polymorphisms with type 2 diabetes mellitus. Exp Mol Med 2008, 40(5):523-532

36. Mannila MN, Lovely RS, Kazmierczak SC, Eriksson P, Samnegård A, Farrell D, Hamsten A, Silveira A: Elevated plasma fibrinogen gamma' concentration is associated with myocardial infarction: effects of variation in fibrinogen genes and environmental factors. J Thromb Haemost 2007, 5(4):766-773.

37. Paffen E, Medina P, de Visser M, van Wijngaarden A, Zorio E, Estellés $A$, Rosendaal FR, España F, Bertina RM, Doggen CJ: The -589C $>T$ polymorphism in the interleukin-4 gene (IL-4) is associated with a reduced risk of myocardial infarction in young individuals. J Thromb Haemost 2008, 6(10):1633-1638

38. Schwender H, Zucknick M, Ickstadt K, Hermann MB, network TG: A Pilot study on the application of statistical classification procedures to molecular epidemiological data. toxicology letters 2004, 151:291-299.

39. Musani SK, Shriner D, Liu N, Feng R, Coffey CS, Yi N, Tiwari HK, Allison DB: Detection of gene $x$ gene interactions in genome-wide association studies of human population data. Hum Hered 2007, 63(2):67-84

40. Kawanishi M, Tamori Y, Okazawa H, Araki S, Shinoda H, Kasuga M: Role of SNAP23 in insulin-induced translocation of GLUT4 in 3T3-L1 adipocytes. Mediation of complex formation between syntaxin4 and VAMP2. J Biol Chem 2000, 275:8240-8247.

41. Gale EA, Gillespie KM: Diabetes and gender. Diabetologia 2001 44(1):3-15.

42. Lin HY, Xu Q, Yeh S, Wang RS, Sparks JD, Chang C: Insulin and leptin resistance with hyperleptinemia in mice lacking androgen receptor. Diabetes 2005, 54:1717-1725.

43. Yeh S, Hu YC, Wang PH, Xie C, Xu Q, Tsai MY, Dong Z, Wang RS, Lee TH, Chang C: Abnormal mammary gland development and growth retardation in female mice and MCF7 breast cancer cells lacking androgen receptor. J Exp Med 2003, 198:1899-1908.

44. Cho YS, Go MJ, Kim YJ, Heo JY, Oh JH, Ban HJ, Yoon D, Lee MH, Kim DJ, Park $\mathrm{M}$, et al: A large-scale genome-wide association study of Asian populations uncovers genetic factors influencing eight quantitative traits. Nat Genet 2009, 41(5):527-534.

45. Vollenweider P: Insulin resistant states and insulin signaling. Clin Chem Lab Med 2003, 41(9):1107-1119.

46. Chang L, Chiang SH, Saltiel AR: Insulin signaling and the regulation of glucose transport. Mol Med 2004, 10(7-12):65-71.

47. Valverde AM, Benito M, Lorenzo M: The brown adipose cell: a model for understanding the molecular mechanisms of insulin resistance. Acta Physiol Scand 2005, 183(1):59-73.

48. Delarue J, Magnan C: Free fatty acids and insulin resistance. Curr Opin Clin Nutr Metab Care 2007, 10(2):142-148.

49. Joachims T: Making large-scale SVM learning practical. Advances in kernel methods - support vector learning. MIT Press; 1999:169-184.

50. Zhou N, Wang L: Effective selection of informative SNPs and classification on the HapMap genotype data. BMC Bioinformatics 2007 8:484

doi: $10.1186 / 1471-2156-11-26$

Cite this article as: Ban et al., Identification of Type 2 Diabetes-associated combination of SNPs using Support Vector Machine BMC Genetics 2010, $11 \cdot 26$

\section{Submit your next manuscript to BioMed Central} and take full advantage of:

- Convenient online submission

- Thorough peer review

- No space constraints or color figure charges

- Immediate publication on acceptance

- Inclusion in PubMed, CAS, Scopus and Google Scholar

- Research which is freely available for redistribution

Submit your manuscript at www.biomedcentral.com/submit
C Biomed Central 